\title{
Habilidades sociales: \\ diferencias entre adolescentes institucionalizados y adolescentes que viven con padres
}

\section{Ireri Yunuen Vázquez García ${ }^{1}$ y Ruth Vallejo Castro ${ }^{1}$}

\section{Resumen}

Las investigaciones y las teorías acerca del desarrollo humano nos dan cuenta de las problemáticas que se presentan en cada período de la vida, siendo de primordial interés en esta investigación la adolescencia. Una de las áreas de mayor dificultad en este período es la del desarrollo de las habilidades sociales y su repercusión en las relaciones interpersonales. En la actualidad, en los medios de comunicación masiva

1 Universidad Michoacana de San Nicolás de Hidalgo, ireriyvg21@gmail.com 
nos enfrentamos con que en nuestra población los adolescentes son víctimas de las redes sociales que los van llevando a tener comportamientos violentos hacia sí mismos, hacia sus compañeros de clases o hacia su propia familia. Ello nos habla de la grave conflictiva que se está presentado a nivel social respecto a poder comprender lo que la otra persona está viviendo y sintiendo, así como de la poca tolerancia que se tiene respecto al otro. Es por ello la importancia de la investigación realizada a este respecto y de la difusión que se realice de la misma. El principal interés de esta investigación fue analizar si existen diferencias significativas en el desarrollo de habilidades sociales como la empatía y la tolerancia a la frustración en dos grupos de adolescentes: el primer grupo está conformado por adolescentes que viven en casas hogar porque han sido retirados de sus hogares de origen por diversas circunstancias y el segundo grupo está conformado por adolescentes que viven con sus padres y que se encuentran estudiando.

Palabras clave: habilidades sociales; adolescencia; institucionalización; casa hogar; familia.

\section{Abstract}

Research and theories about human development give us an account of the problems that arise in each period of life. In this research, the primary interest is adolescence. One of the areas of greatest difficulty in 
this period is the development of social skills and its impact on interpersonal relationships. Currently, in the mass media we are faced with the fact that adolescents in our population are victims of social networks that lead them to violent behavior towards themselves, their classmates or their own family. This tells us about the serious conflict that is occurring at a social level regarding being able to understand what the other person is living and feeling, as well as the little tolerance that one has regarding the other. That is why the importance of the research carried out in this regard and its dissemination is important. The aim of this study is to analyze whether there are significant differences in the development of social skills such as empathy and tolerance of frustration in two groups of adolescents: the first group is made up of adolescents who live in family homes because they have been withdrawn from their homes of origin due to various circumstances and the second group is made up of adolescents who live with their parents and who are studying.

Keywords: social skills; adolescence; institutionalization; foster home; family.

Abordando el tema de la adolescencia, Casas y Ceñal (2005) mencionan que los adolescentes se pueden ver enfrentados a obstáculos que impiden el disfrute de este período como los siguientes: "restricción de movimiento y libertad, falta de responsabilidad, proble- 
mas de sexualidad e intimidad, aislamiento de los modelos adultos y falta de poder y control" (p. 22). También mencionan que es justo en este momento en donde se encuentran en búsqueda del desarrollo de sus habilidades y capacidades, por eso es que se ven involucrados en situaciones en las que pueden poner en práctica qué tan competentes pueden llegar a ser, al mismo tiempo que se encuentran en la búsqueda constante de la independencia de la familia, de la aceptación dentro de algún grupo, así como de la búsqueda de la identidad y de una imagen propia.

De este período existen muchas clasificaciones, sin embargo nos enfocaremos a la realizada por Casas y Ceñal (2005) que la dividen en tres: adolescencia temprana (11-13 años), adolescencia media (14-17 años) y adolescencia tardía (17-21 años), mencionando algunas características distintivas de cada una: (a) adolescencia temprana, en la que comienzan a presentarse los caracteres sexuales secundarios, de la misma forma comienzan los contactos con el sexo opuesto, se encuentra presente el reclamo por su intimidad e independencia, su pensamiento aún es concreto, no tienen capacidad para ver las consecuencias de sus actos, su orientación es narcisista y suelen ser muy egoístas; (b) adolescencia media, cuando el pensamiento aún se encuentra entre lo concreto y lo abstracto, comienzan a desarrollar la capacidad de saber las consecuencias de lo que hacen, se consideran omnipotentes e invulnerables, se encuentran luchando por obtener el control de su vida, 
las opiniones de los amigos son más importantes que las de los padres, y comienzan a presentarse las fantasías románticas; (c) adolescencia tardía, cuando el pensamiento ya es completamente abstracto, un tiempo más estable.

En este período también se experimentan duelos sumamente importantes para el desarrollo de la identidad del adolescente, Aberastury y Knobel (2012) mencionan que son tres. Primero, el duelo por el cuerpo infantil: el adolescente se da cuenta que se está perdiendo el cuerpo infantil, que aún se tiene una mente infantil y que se está empezando a desarrollar un cuerpo adulto lo cual le genera un sentimiento de impotencia. Segundo, el duelo por la identidad y el rol infantil: aquí el adolescente termina dejando a cargo de los padres la mayor parte de las obligaciones y responsabilidades ya que se encuentra en una constante confusión de roles; por un lado no puede continuar con la dependencia infantil que tenía pero tampoco puede ser totalmente independiente como un adulto. Tercero, el duelo por los padres de la infancia: se presentan contradicciones del pensamiento que van a provocar rupturas en la comunicación con sus padres y al mismo tiempo se da la sustitución de las figuras parentales internalizadas por figuras idealizadas, pero este duelo se dificulta aún más porque también los padres tienen que elaborar la pérdida por el hijo que está dejando de ser niño para convertirse en adulto.

Como señalan Samper, Mestre, Malonda y Mesurado (2015), durante 
[...] la adolescencia las relaciones sociales con los compañeros adquieren una especial importancia por la relevancia que tienen para el adolescente y también por el impacto que estas relaciones tienen en el ajuste psicosocial de la persona. En el contexto escolar la aceptación o rechazo social por los iguales están relacionados con un mayor o menor equilibrio emocional y en general con la adaptación de los adolescentes". (p. 849)

Con lo anteriormente señalado, queda claro que para el adolescente es de suma importancia la aceptación por parte de los grupos en los que busca la socialización, ya que a través de ellos logra el desarrollo de habilidades y recursos, y encuentran apoyo, lo que contribuye a su bienestar emocional.

En la adolescencia también se presentan otros fenómenos como los estudiados por Aguilar, Valdez, González, Rivera, Carrasco, Gómora, Pérez y Vidal (2015) en su investigación Apatía, desmotivación, desinterés, desgano y falta de participación en adolescentes mexicanos. En ella, se menciona que las cuestiones académicas y laborales de la actualidad impactan negativamente a los más jóvenes, provocando en ellos desmotivación, insatisfacción y apatía. Algunas cuestiones como "escribir, hacer tareas, ir a la escuela, ayudar en casa, lavar trastes, levantarse temprano, recoger su cuarto, estar en casa, hacer deberes, ir a la tienda, no hacer nada, ver televisión, correr y jugar futbol" (p. 329) causan apatía en los 
adolescentes. En el aspecto de la desmotivación encontraron que lo que más desmotiva a los adolescentes es la "tarea, peleas con amigos, enfermedad, que no los apoyen, quehaceres domésticos, deportes, problemas familiares, fracaso, que algo salga mal, no poder hacer lo que quiere, no ser alguien en la vida y la crítica de la sociedad" (p. 329).

En este período es de suma importancia que se desarrollen habilidades sociales tales como la empatía, la tolerancia a la frustración, la asertividad, la escucha, el respeto, la compasión, la regulación emocional, entre otras, que le ayudarán al adolescente a relacionarse de una manera más saludable con las personas que le rodean.

\section{Empatía}

La empatía, ha sido estudiada por muchos autores desde diferentes perspectivas. Actualmente, se manejan tanto la perspectiva cognitiva como la afectiva. La fantasía, entendida como tendencia a identificarse con personajes de ficción, y la adopción de perspectivas se encontrarían en la dimensión cognitiva. En la dimensión afectiva tendríamos la angustia empática, entendida como tendencia a experimentar sentimientos de compasión y preocupación por el otro, así como la aflicción personal, una ansiedad que se experimenta al ser testigo de una situación desagradable para el otro (Fernández, López y Márquez, 2008). Es a partir de los años noventa que comienza a estudiarse la empatía desde la perspectiva de 
la Inteligencia Emocional. En este sentido, Salovey y Mayer (citados en Fernández et al, 2008), entienden que la inteligencia emocional se encuentra constituida por cuatro capacidades: percepción, facilitación, comprensión y regulación emocional.

Desde la perspectiva neurofisiológica, Fernández y cols. (2008) han encontrado que "la corteza prefrontal parece ser la principal área implicada en el procesamiento de la empatía y la regulación del procesamiento empático: la región frontal dorsolateral estaría más especializada en la empatía cognitiva, mientras que la región orbitofrontal lo estaría en la empatía afectiva" (p. 289). Pero mencionan que en realidad son muchas más las áreas implicadas ya que la empatía se conforma de diversos componentes y cada uno de esos componentes se encuentran ubicados en diferentes áreas. Desde el ámbito clínico, partiendo del DSM-IV-TR, "se puede comprobar que la carencia de empatía es una de las características centrales del trastorno del desarrollo denominado síndrome de asperger" (Fernández et al., 2008, p. 291), a quien Gillbert (1990) denominó como trastorno de empatía. Así, el criterio de déficit de la empatía puede ser tomado en cuenta como parte de la evaluación de algunos trastornos de personalidad como el trastorno de personalidad esquizoide, el trastorno narcisista, el trastorno antisocial, el trastorno límite y los del espectro autista.

Eisenberg (2000) entiende la empatía en términos de respuesta afectiva que posibilita comprender la situación emocional del otro, una condición básica para el 
desarrollo. Repetto (citado en Rey, 2003) menciona que la empatía "es una habilidad social de alto nivel, que hace referencia a la capacidad de comprender los sentimientos de otra persona" (p. 186). También menciona que es "una habilidad que media la presencia de diferentes formas de conducta prosocial, por lo que su desarrollo es indispensable para que los individuos presenten comportamientos prosociales en general", p. 186, (Fuentes, 1989, Loeber, Hay, 1997, citados en Rey, 2003). Fuentes, aplicó a 170 niños el Índice de Empatía y Ansiedad, encontrando que la empatía se ubicaba más elevada en los adolescentes que en los niños, y ello lo llevó a concluir que la cognición es sumamente importante en el desarrollo de esta habilidad empática, (citado en Rey, 2003). Otras investigaciones arrojan que los varones tienen menos capacidad empática y son más proclives a presentar una conducta antisocial; de la misma forma que muestran menos empatía, respeto y sociabilidad, mayores sentimientos de soledad, más conductas agresivas y mayor capacidad de liderazgo.

En el mismo sentido, Sánchez, Oliva y Parra (2006), hacen la diferencia entre empatía disposicional y empatía situacional, en donde la primera hace referencia a la empatía como un rasgo de personalidad y la segunda hace referencia a sentir empatía dependiendo de la situación que se experimenta. De la misma manera, Oliva, Antolín, Pertegal, Ríos, Parra, Hernando, y Reina, (2011), clasifican la empatía también como disposicional y situacional, pero agregan la afectiva que es la capacidad de compartir un sentimiento adecuado al estado 
emocional del otro (sentir), y la cognitiva que es la capacidad de comprender al otro (darse cuenta). Un aspecto importante que influye en el desarrollo de la empatía son los estilos de crianza ya que Davis (citado en Sánchez et al., 2006) menciona que "quienes sienten satisfechas sus necesidades emocionales en una familia con vínculos seguros y afectuosos, estarán menos inquietos por sus propias preocupaciones y podrán interesarse y ser sensibles a las necesidades de los demás; quienes crecen en un ambiente de amor y afecto tendrán un buen modelo que adoptar sobre cómo actuar con los demás" (p. 262). Asimismo, se menciona que los adolescentes de culturas tradicionales, en las cuales tienen que contribuir con todo lo relacionado con la familia cuentan con mayor prosocialidad, a diferencia de los adolescentes de las culturas en donde lo importante es la competencia y la autonomía.

En su investigación, Sánchez et al. (2006), encontraron que los niveles de empatía se van incrementando durante la adolescencia pero sólo en el caso de las chicas, es decir, que ellas son significativamente más empáticas y esta característica junto con la prosocialidad se encuentran relacionadas con las variables de familia y de intimidad con el/la mejor amigo/a. En el caso de los chicos los que puntuaron más altos en empatía fueron los que se encontraban en ambientes adaptables, cohesionados, afectuosos, en donde se tenía un control de la conducta, que se podía hablar frecuentemente entre ellos y en los cuales existían pocos conflictos; los resultados de 
esta investigación refuerzan lo anteriormente dicho por Davis.

Martínez-Otero (2011), coincide con los autores anteriores en el sentido de que en la empatía se encuentran tanto aspectos cognitivos como emocionales, de la misma manera se fundamenta lo anteriormente dicho con una investigación que realizó con estudiantes universitarios en donde encontró que los varones puntuaban más altos en la vertiente cognitiva que en la afectiva, es decir, que los varones tienen más capacidad para comprender los estados emocionales de los demás y menos capacidad en involucrase en situaciones afectivas externas.

\section{Tolerancia a la frustración}

La frustración comenzó a estudiarse desde el año 1950, con la intención de demostrar algunas hipótesis que se planteaban en ese tiempo, como, por ejemplo, que "la frustración generaba agresión, fijación, regresión y conflicto" Yates (citado en Kamenetzky, Cuenya, Elgier, López Seal, Fosacheca, Martín, y Mustaca, 2009, p. 193), así como que esta misma puede generar fobias, enfermedades, depresiones o propensión a desarrollar algún tipo de adicción o a buscar ciertos tipos de riesgos. En este sentido Rosabal, Romero, Gaquín y Hernández (2015) mencionan que existen algunos rasgos de personalidad en los adolescentes que hacen que busquen o lleven a cabo conductas de riesgo; dentro de esos rasgos se encuentra por ejemplo 
el poco control de impulsos que se relaciona con la baja tolerancia a la frustración.

En investigaciones con adolescentes como la de Ulloa, Contreras, Paniagua y Victoria (2013), se ha estudiado la frecuencia de autolesiones y características clínicas en adolescentes de la Ciudad de México, encontrando que dentro de los motivos que los adolescentes tienen para autolesionarse está la baja tolerancia a la frustración y manipulación en el $42.1 \%$ de los casos. Otro estudio que aborda el tema del maltrato infantil, realizado por Santana, Sánchez y Herrera (1998), señala que la madre es la figura parental que más agrede y dan una serie de características de esta figura entre las cuales está la baja tolerancia a la frustración. De igual forma, Casullo (2004) realiza una investigación sobre ideación y conducta suicida en adolescentes, en la cual menciona que uno de los comportamientos que se encuentran ligados con las ideaciones y el comportamiento suicida es el impulsivo, que se caracteriza por la baja tolerancia a la frustración.

De acuerdo con lo encontrado en las anteriores investigaciones, queda clara la importancia que tiene el desarrollo de la tolerancia a la frustración como una habilidad básica en el adolescente, ya que el no desarrollo de esta habilidad tendrá repercusiones trascendentales en la vida del adolescente y de las personas que lo rodean, tanto en el presente como en el futuro. Por el contrario, si se trabaja en el desarrollo de esta y otras habilidades, los resultados se verán 
reflejados en una sociedad más tolerante y más empática con su contexto.

Se decide trabajar con este período del desarrollo justo porque es uno de los más vulnerables, ya que es un período en el cual se presentan muchos cambios respecto a lo físico, lo cognitivo y lo psicológico entre muchos otros, principalmente en los adolescentes que han crecido y se encuentran viviendo en casas hogar alejados de su familia de origen

\section{Método}

El diseño de este estudio es cuantitativo, de alcance descriptivo. Se trabajó con la medición de la empatía y la tolerancia a la frustración en 68 adolescentes que se encuentran en casas hogar y con 68 adolescentes que se encuentran viviendo con sus padres, así como estudiando la preparatoria y la universidad.

En ambos grupos se aplicó la Escala Básica de Empatía, que fue traducida y adaptada de la Basic Empathy Scale de Jolliffe y Farrington, que originalmente incluía 20 ítems y después de la adaptación solamente quedaron 9 ítems para medir el nivel de empatía afectiva, cognitiva y global. Esta escala fue adaptada por Oliva, Antolín, Pertegal, Ríos, Parra, Hernando, y Reina (2011) en población española.

También se aplicó la Escala para la Evaluación de la Tolerancia a la Frustración, la cual es una adaptación española de la subescala Stress Management del Emocional Quotient Inventory (EQ-i, YV) de Bar-On y 
Parker (2000). La escala está conformada por 8 items que miden el factor de manejo del estrés, en el cual se encuentran la tolerancia al estrés y el control de impulsos. Ambas escalas fueron obtenidas del libro de Oliva et al. (2011).

El análisis de resultados se llevó cabo con el apoyo del programa estadístico SPSS. Se analizó la distribución de los participantes de casas hogar y de los que viven con sus padres en relación con los niveles de empatía afectiva, empatía cognitiva, empatía global y tolerancia a la frustración en la que sus puntuaciones los ubicaron.

Posterior a la aplicación de las escalas y al análisis de resultados se implementó un taller para trabajar la empatía y la tolerancia a la frustración con los adolescentes de casas hogar.

\section{Resultados}

En cuanto a los resultados con respecto a la Empatía Afectiva en los adolescentes de casas hogar, 31 participantes (46\%) muestran tener una empatía afectiva muy baja; 14 (21\%) se ubican en la categoría baja; 7 (10\%) en la categoría media; otros 7 se ubican en el nivel de empatía afectiva alta y los 9 restantes (13\%) muestran una empatía afectiva muy alta.

Respecto a los adolescentes que viven con sus padres, 47 participantes (69\%) muestran tener una empatía afectiva muy baja; $7(10 \%)$ se ubican en la categoría baja; 6 (9\%) se ubican en la categoría media; 
$4(6 \%)$ en el nivel de empatía afectiva alta y los 4 restantes (6\%) muestran una empatía afectiva muy alta.

Con respecto a la Empatía Cognitiva en adolescentes de casa hogar, se obtuvieron los siguientes resultados: 30 participantes (44\%) se ubican en la categoría de muy baja; 14 (21\%) en una empatía cognitiva baja; 8 (12\%) se ubican en el nivel medio, 9 (13\%) en una empatía cognitiva alta y los 7 restante (10\%) en una empatía cognitiva muy alta.

En cuanto a los adolescentes que viven con sus padres, 29 participantes (42\%) se ubican en la categoría de muy baja; 11 (16\%) cuentan con una empatía cognitiva baja; 8 (12\%) se ubican en un nivel medio; 12 (18\%) cuentan con una empatía cognitiva alta y los 8 restantes (12\%) manifiestan una empatía cognitiva muy alta.

Con respecto a la empatía total, la cual comprende la empatía afectiva y la empatía cognitiva, se pueden observar los siguientes resultados en los adolescentes de casas hogar: 36 de los participantes (53\%) muestran una empatía muy baja; 11 (16\%) se ubican en la empatía baja; 8 (12\%) muestran un nivel medio; 8 (12\%) la expresan alta y los 5 restantes (7\%) la tienen muy alta.

En los adolescentes que viven con sus padres se encontró lo siguiente: 46 de los participantes (68\%) muestran una empatía muy baja; 7 (10\%) se ubican en la empatía baja; 5 (7\%) muestran un nivel medio; 8 
(12\%) la expresan alta y los 2 restantes (3\%) tendrían una empatía total muy alta.

En cuanto a la tolerancia a la frustración, de los adolescentes que viven en casas hogar, 32 participantes (47\%) tienen muy baja tolerancia; $14(21 \%)$ la tienen baja; $7(10 \%)$ la tienen media; otros $7(10 \%)$ la tienen alta y los 8 restantes (12\%) la tienen muy alta.

Con los adolescentes que viven con sus padres los resultados fueron los siguientes: 20 participantes (29\%) tienen muy baja tolerancia; 12 (18\%) la tienen baja; 6 (9\%) la tienen media; 12 (18\%) la tienen alta y $18(26 \%)$ la tienen muy alta.

\section{Discusión}

Los participantes de ambos grupos muestran tener dificultades en la empatía afectiva; es decir, se les dificulta el poder sentir la condición o estado emocional de otra persona (Eisenberg, 2000), así como compartir un sentimiento adecuado al estado emocional del otro. De igual forma, demostraron tener dificultad en la habilidad de comprender al otro, lo cual corresponde a la empatía cognitiva. De la misma manera la mayoría de los adolescentes participantes muestran tener una empatía global muy baja o baja, es decir, que tienen dificultad tanto para sentir con los otros como para comprenderlos (Martínez-Otero, 2011).

Respecto a la tolerancia a la frustración, se encontraron diferencias entre ambos grupos. En el grupo de los adolescentes de casa hogar se encontró 
que el $68 \%$ se encuentran dentro de la categoría de "muy baja" y "baja", por lo que se puede decir que en este grupo hasta este momento no se ha desarrollado esta habilidad. Es posible que esto se deba a la situación de vulnerabilidad en la que se encuentran estos adolescentes y a la complejidad del contexto en el que viven. En cuanto al grupo de los adolescentes que viven con sus padres, estos se encuentran distribuidos en los extremos, es decir, el $47 \%$ se encuentran en las categorías de "muy baja y baja" y el $44 \%$ se encontraron en las categorías de "muy alta y alta", y solo el $9 \%$ se encuentra dentro de la categoría "media" lo que significa que casi la mitad de los adolescentes de este grupo aún no desarrollan esta habilidad.

\section{Conclusiones}

No se encontraron diferencias significativas entre ambos grupos. Ello podría deberse a que, dada la etapa vital en la que se encuentran, los adolescentes aún están en proceso de desarrollar estas habilidades. Por lo anterior, se sugiere trabajar con los adolescentes en el desarrollo de habilidades como la empatía, la tolerancia a la frustración, la asertividad, la escucha, el respeto, la compasión, la regulación emocional. Este tipo de trabajo les permitirá relacionarse de una manera más saludable con las personas que los rodean, incluyendo tanto su sistema familiar como otros grupos sociales porque ciertamente es un período en el cual la 
dificultad en la comunicación y las relaciones interpersonales puede generarles muchos conflictos.

\section{Referencias}

Aberastury, A. y Knobel, M. (2012). La adolescencia normal. México: Ediciones Paidós. Aguilar, Y., Valdez, J., González, N., Rivera, S., Carrasco, C., Gómora, A., Pérez, A., y Vidal, S. (2015). Apatía, desmotivación, desinterés, desgano y falta de participación en adolescentes mexicanos. Enseñanza e Investigación en Psicología, 20(3), 326-336. Recuperado de http:// www.redalyc.org/articulo.oa?id=29242800010

Casas, J. y Ceñal, M. (2005). Desarrollo del adolescente. Aspectos físicos, psicológicos y sociales. Pediatría Integral, 9(1), 20-24.

Recuperado de http://www.sld.cu/galerias/pdf/sitios/puericultura/de sarrollo adolescente(2).pdf

Casullo, M. (2004). Ideaciones y comportamientos suicidas en adolescentes: una urgencia social. XII Anuario de Investigaciones, 173-182.

Recuperado de http://www.scielo.org.ar/pdf/anuinv/v12/v12a17.pdf Eisenberg, N. (2000). Emotion, regulation and moral development. Annual Review of Psychology, 51, 665-697. doi: 10.1146/annurev.psych.51.1.665. 
Fernández, I., López, B. y Márquez, M. (2008).

Empatía: Medidas, teorías y aplicaciones en

revisión. Anales de Psicología, 24(2), 284-298.

Recuperado de

http://www.um.es/analesps/v24/v24_2/12-24_2.pdf

Kamenetzky, V., Cuenya, L., Elgier, M., López Seal, F.,

Fosacheca, S., Martín, L. y Mustaca, E. (2009).

Respuestas de Frustración en Humanos. Terapia

Psicológica, 27(2), 191-201. doi: 10.4067/S0718-

48082009000200005

Martínez-Otero, V. (2011). La empatía en la educación:

estudio de una muestra de alumnos universitarios.

Revista electrónica de Psicología Iztacala, 14(4),

174-190. Recuperado de

http://www.medigraphic.com/pdfs/epsicologia/epi2011/epi114j.pdf

Merino, C. y Grimaldo, M. (2015). Validación estructural de la Escala Básica de Empatía (Basic Empathy Scale) modificada en adolescentes: un estudio preliminar. Revista Colombiana de Psicología, 24(2), 261-270. doi: 10.15446/rcp.v24n2.42514.

Oliva, A., Antolín, L., Pertegal, M., Ríos, M., Parra, A., Hernando, A. y Reina, C. (2011). Instrumentos para la evaluación de la salud mental y el desarrollo positivo adolescente y los activos que lo promueven. Sevilla: Junta de Andalucía. Recuperado de http://www.formajoven.org/AdminFJ/doc_recursos/ 201241812465364.pdf 
Rey, C. (2003). La medición de la empatía en preadolescentes y adolescentes varones: adaptación y validación de una escala. Revista Latinoamericana de Psicología, 35(2), 185-194.

Recuperado de http://www.redalyc.org/articulo.oa? id=80535206

Rosabal, E., Romero, N., Gaquín, K. y Hernández, R. (2015). Conductas de riesgo en los adolescentes. Revista Cubana de Medicina Militar, 44(2), 218229. Recuperado de http://scielo.sld.cu/pdf/mil/v44n2/mil10215.pdf Samper, P., Mestre, V., Malonda, E. y Mesurado, B. (2015). Victimización en la escuela: relación de la crianza y variables funcionales-disfuncionales del desarrollo. Anales de Psicología, 31(3), 849-858. doi: 10.6018/analesps.31.3.173291

Sánchez-Queija, I., Oliva, A. y Parra, A. (2006).

Empatía y conducta prosocial durante la adolescencia. Revista de Psicología Social, 21(3), 259-271. Recuperado de https://personal.us.es/oliva/prosocial.pdf Santana, R., Sánchez, R. y Herrera, E. (1998). El maltrato infantil: un problema mundial. Salud Pública de México, 40(1), 58-65. Recuperado de http://saludpublica.mx/index.php/spm/article/view/ $\underline{6058 / 7061}$

Ulloa, R., Contreras, C., Paniagua, K. y Victoria, G. (2013). Frecuencia de autolesiones y características clínicas asociadas en adolescentes 
que acudieron a un hospital psiquiátrico infantil. Salud Mental, 36(5), 417-420. Recuperado de http://www.scielo.org.mx/pdf/sm/v36n5/v36n5a10. pdf

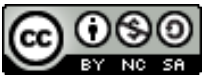

Esta obra está bajo una

\section{Licencia Creative Commons Atribución-NoComercial-} Compartirlgual 4.0 Internacional

Usted es libre de compartir o adaptar el material en cualquier medio o formato bajo

las condiciones siguientes: (a) debe reconocer adecuadamente la autoría, proporcionar un enlace a la licencia e indicar si se han realizado cambios; (b) no puede utilizar el material para una finalidad comercial y (c) si remezcla, transforma

o crea a partir del material, deberá difundir sus contribuciones bajo la misma licencia que el original.

Resumen de la licencia https://creativecommons.org/licenses/by-nc-sa/4.0/deed.es_ES

Texto completo de la licencia https://creativecommons.org/licenses/by-nc-sa/4.0/legalcode 